\title{
Prevalence and real-world management of NSTEMI with multivessel disease
}

\author{
Angus A. W. Baumann ${ }^{1,2} \wedge$, Rosanna Tavella ${ }^{1,3,4}$, Tracy M. Air ${ }^{3,4}$, Aashka Mishra ${ }^{1}$, Nicholas J. Montarello ${ }^{1}$, \\ Margaret Arstall ${ }^{3,5}$, Chris Zeitz ${ }^{1,3}$, Matthew I. Worthley ${ }^{1,3}$, John F. Beltrame ${ }^{1,3,4}$, Peter J. Psaltis ${ }^{1,3,6}$ \\ ${ }^{1}$ Department of Cardiology, Central Adelaide Local Health Network (CALHN), Adelaide, Australia; ${ }^{2}$ Department of Medicine, Alice Springs \\ Hospital, Alice Springs, Australia; ${ }^{3}$ Adelaide Medical School, The University of Adelaide, Adelaide, Australia; ${ }^{4}$ Basil Hetzel Institute for Translational \\ Health Research, The Queen Elizabeth Hospital, Woodville South, Australia; ${ }^{5}$ Northern Adelaide Local Health Network (NALHN), Adelaide, \\ Australia; ${ }^{6}$ Vascular Research Centre, Lifelong Health Theme, South Australian Health \& Medical Research Institute, Adelaide, Australia \\ Contributions: (I) Conception and design: AAW Baumann, R Tavella, TM Air, A Mishra, JF Beltrame, PJ Psaltis; (II) Administrative support: R \\ Tavella, TM Air, M Arstall, C Zeitz, MI Worthley, JF Beltrame; (III) Provision of study materials or patients: R Tavella, TM Air, M Arstall, C Zeitz, \\ MI Worthley, JF Beltrame; (IV) Collection and assembly of data: AAW Baumann, R Tavella, TM Air, A Mishra, NJ Montarello; (V) Data analysis \\ and interpretation: AAW Baumann, R Tavella, TM Air, A Mishra, JF Beltrame, PJ Psaltis; (VI) Manuscript writing: All authors; (VII) Final approval \\ of manuscript: All authors. \\ Correspondence to: A/Prof. Peter J. Psaltis. Vascular Research Centre, Lifelong Health Theme, South Australian Health \& Medical Research Institute, \\ North Terrace, Adelaide, South Australia, Australia. Email: peter.psaltis@sahmri.com; peter.psaltis@adelaide.edu.au.
}

Background: Non-ST elevation myocardial infarction (NSTEMI) has higher post-discharge mortality than ST-elevation myocardial infarction (STEMI). Prognosis worsens in those with multivessel coronary disease (MVD). However, information about the prevalence and extent of MVD in NSTEMI is limited, in turn limiting insights into optimal treatment strategies. This study aimed to define the prevalence and extent of MVD, preferred treatment strategies and the predictors of MVD in a real-world NSTEMI population.

Methods: The Coronary Angiogram Database of South Australia (CADOSA) was used to identify consecutive patients presenting to major teaching hospitals with NSTEMI between 2012 and 2016. Obtaining clinical and angiographic details, patients were stratified by the number of significantly diseased vessels $(0,1,2,3-\mathrm{VD})$, defined by a stenosis of $\geq 70 \%$, or $\geq 50 \%$ in the left main coronary artery. Data was analysed retrospectively.

Results: The prevalence of MVD (2- or 3-VD) was 42\% amongst 3,722 NSTEMI presentations. Multivariate logistic regression modelling showed age, male gender, diabetes, dyslipidaemia and prior myocardial infarction predicted MVD over 1-VD or 0-VD. Percutaneous coronary intervention (PCI) was performed in $42 \%$ of patients with MVD. This comprised $61 \%$ of $2-\mathrm{VD}$ patients and only $22 \%$ of $3-\mathrm{VD}$ patients, with $24 \%$ and $66 \%$ of each group referred for coronary bypass grafting, respectively. Among MVD patients treated with PCI, 76\% had their culprit lesion treated alone in the index admission.

Conclusions: In this NSTEMI cohort, over $40 \%$ had MVD. Notably, a minority of patients with MVD undergoing PCI received multivessel revascularisation. This real-world practice emphasises that further evaluation is required to determine whether complete revascularisation is beneficial in NSTEMI, as reported for STEMI.

Keywords: Non-ST elevation myocardial infarction (NSTEMI); multivessel coronary artery disease; coronary artery bypass grafting; percutaneous coronary intervention; coronary revascularisation

^ ORCID: 0000-0003-4128-7012. 
Submitted Aug 18, 2021. Accepted for publication Jan 13, 2022.

doi: $10.21037 / \mathrm{cdt}-21-518$

View this article at: https://dx.doi.org/10.21037/cdt-21-518

\section{Introduction}

Although the overall incidence of acute myocardial infarction (AMI) has declined in many developed countries over recent decades $(1,2)$, the incidence of non-ST-elevation myocardial infarction (NSTEMI) in comparison to STelevation myocardial infarction (STEMI) continues to increase (3). This has important implications as NSTEMI carries a greater long-term mortality burden after hospital discharge than STEMI $(4,5)$. Among patients suffering AMI, the presence of multivessel coronary artery disease (MVD) also confers a poor prognosis $(6,7)$. However, information about the prevalence and predictors of MVD in NSTEMI is surprisingly limited compared to STEMI and stable coronary artery disease (CAD) cohorts $(8,9)$, as are guidelines relating to the optimal management of nonculprit coronary lesions $(10,11)$. This study aims to define the prevalence and key clinical predictors of MVD in a large Australian cohort of consecutive NSTEMI patients undergoing invasive coronary angiography and define the mode and pattern of revascularisation in these patients. We present the following article in accordance with the STROBE reporting checklist (available at https://cdt. amegroups.com/article/view/10.21037/cdt-21-518/rc).

\section{Methods}

\section{Study design, population and variables}

This study is based upon the Coronary Angiogram Database of South Australia (CADOSA) which captures all patients undergoing coronary angiography in participating institutions. It is based on the National Cardiovascular Data Registry (NCDR) CathPCI registry, using the same data definitions including for NSTEMI and each traditional cardiovascular risk factor (12). NSTEMI was defined as a typical rise and fall in cardiac biomarkers with ST-segment depression or t-wave abnormalities and/or ischaemic symptoms. Dyslipidemia was defined as a documented history of dyslipidemia diagnosed and/or treated by a physician. Hypertension was defined as a history of hypertension being actively treated, a blood pressure greater than $140 \mathrm{mmHg}$ systolic or $90 \mathrm{mmHg}$ diastolic or current use of anti-hypertensive pharmacologic therapy.
Smoking was defined as a confirmed history of smoking at any time in the past. Diabetes was defined as a documented history of diabetes including a need for anti-diabetic agents, or a fasting blood sugar $>7 \mathrm{mmol} / \mathrm{L}$. Family history was defined as any blood relative with a history of angina, AMI or sudden cardiac death at an age less than 55 years.

CADOSA is endorsed by the American College of Cardiology. Data is collected prospectively, in real time, by trained data officers ensuring high quality data. The primary role of these data officers is collecting and maintaining the registry data at their respective sites. Audits are undertaken on the registry data. Data collection is performed using a standard data collection form and guided with extensive data specifications. The registry data elements and specifications are compatible with the American College of Cardiology CathPCI registry. Individual consent for this retrospective analysis was waived and the study was approved by the Central Adelaide Local Health Network Human Ethics Committee (HREC reference number HREC/15/ TQEH/252). The study was conducted in accordance with the Declaration of Helsinki (as revised in 2013).

This study included consecutive patients presenting with NSTEMI to three tertiary hospitals in Adelaide, South Australia, between the $1^{\text {st }}$ of January 2012 and the $31^{\text {st }}$ of December 2016, captured by CADOSA. For patients who had undergone multiple admissions and coronary angiograms during the recruitment window, only the first visit was used. Patients with a history of previous coronary artery bypass grafting (CABG) were excluded from this study. The selection of patients is summarized in Figure 1.

Patient characteristics, including traditional risk factors for atherosclerosis, were extracted from the clinical record. Patients were grouped by the number of coronary arteries with a significant stenosis, defined as a lesion of angiographic severity $\geq 50 \%$ in the left main coronary artery (LM) or $\geq 70 \%$ in the left anterior descending (LAD), left circumflex (LCx) or right coronary artery (RCA) systems. Lesion severity was determined by experienced operators using visual assessment and all lesions were described quantitatively as part of standard clinical practice. Onevessel disease (1-VD) was defined as significant narrowing in one of the LAD, LCx or RCA or its first-order branches. Two-vessel disease (2-VD) was defined as significant 


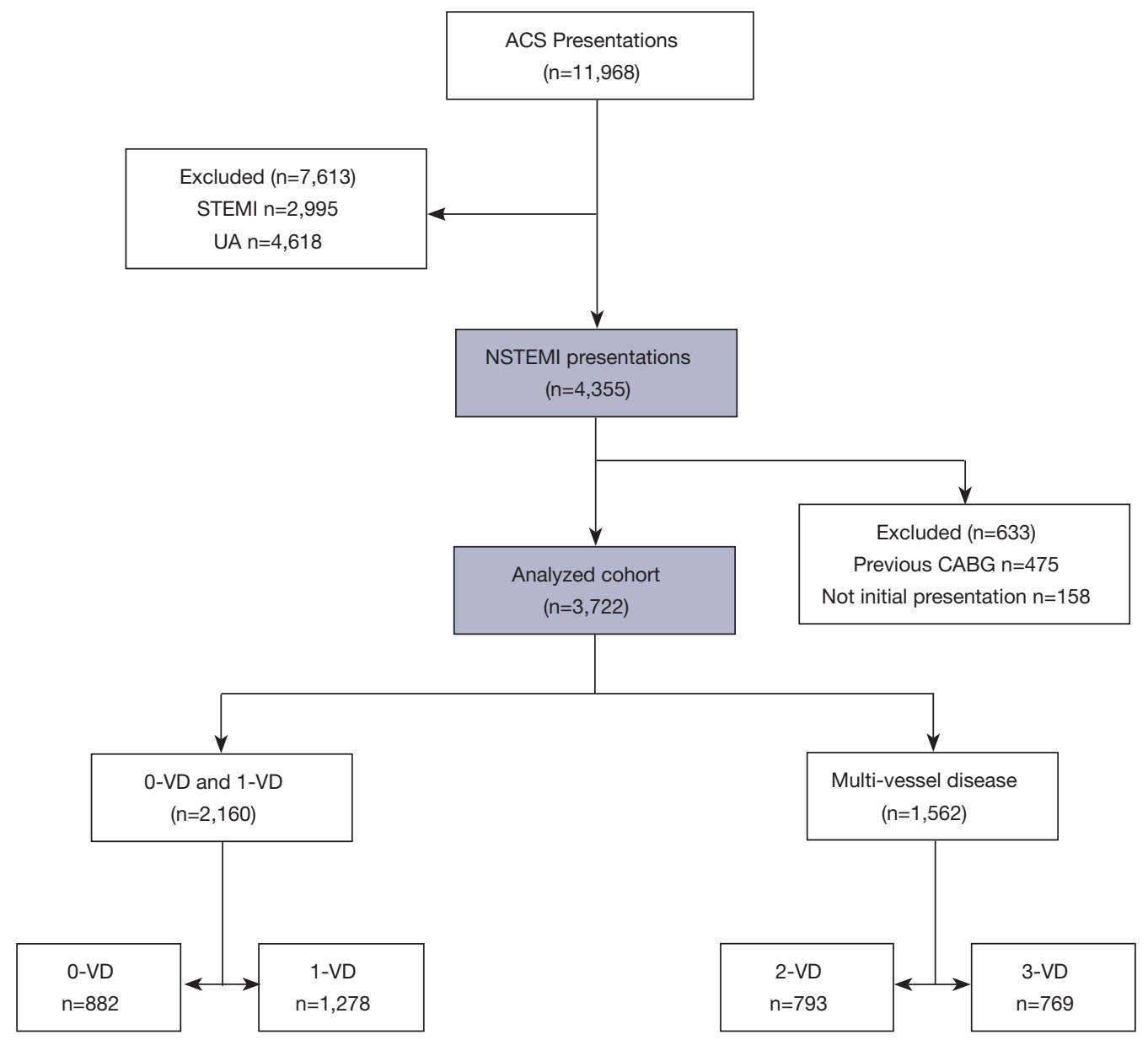

Figure 1 Patient selection flow diagram. This flow diagram shows the number of NSTEMI presentations screened, how many were included in the final analysis, and the number of presentations in each analysed group. NSTEMI, non-ST elevation myocardial infarction. $\mathrm{VD}$, vessel disease; CABG, coronary artery bypass grafting.

narrowing in the LM, or two of the LAD, LCx and RCA. Three-vessel disease (3-VD) was defined as significant narrowing in the $L M$ and the RCA, or in each of the LAD, LCx and RCA. Zero-vessel disease (0-VD) was defined as no coronary lesions satisfying the above criteria. Information about the location and severity of all coronary artery lesions was collected, along with the recommended treatment plan. This was classified as medical management only, percutaneous coronary intervention (PCI), CABG or "indeterminate", which encompassed any treatment not readily categorised into the other three groups, including deferral of treatment pending functional ischaemic testing, myocardial viability assessment or multi-disciplinary team discussion. Where patients went on to receive PCI, the number of treated vessels during the index admission or within 90 days of discharge were recorded. Finally, the incidence of in-hospital mortality was also determined.

\section{Statistical analysis}

Continuous data were tested for normality of distribution and are presented as mean $\pm \mathrm{SD}$ or median and range. One-way ANOVA was used for comparison of continuous variables and Chi-square tests for categorical variables. Two-sided $\mathrm{P}$ values $<0.05$ were considered to indicate statistical significance. To identify clinical predictors of MVD in NSTEMI patients, all statistically significant variables from the univariate analyses were entered into a logistic regression model. In an iterative process, covariates were removed if they were non-significant and a non-confounder. Confounding was defined as a change in any remaining parameter estimate of $15 \%$ or greater. At the end of this iterative process, the model contained 
Table 1 Characteristics of NSTEMI patients categorised by number of coronary arteries containing significant angiographic disease

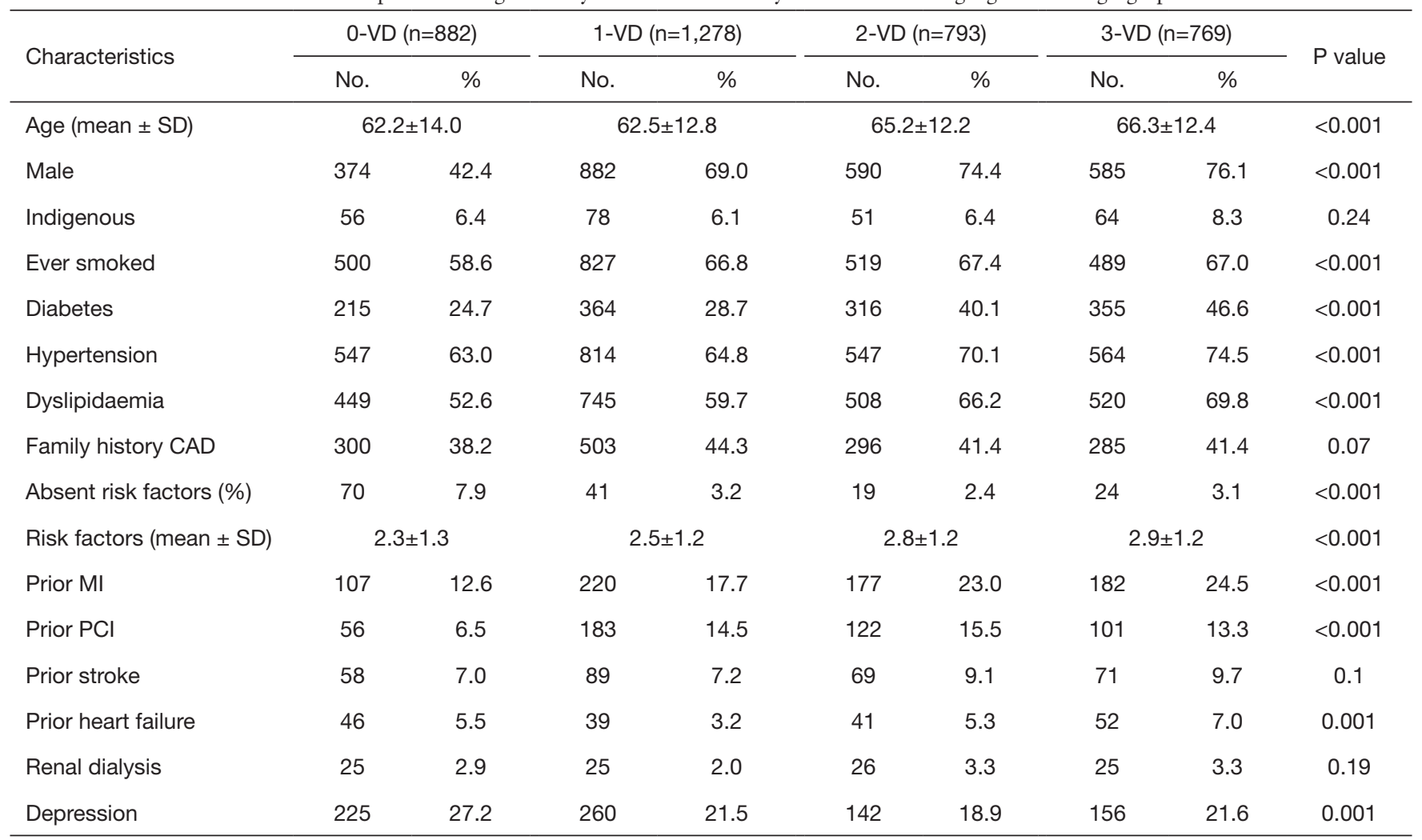

NSTEMI, non-ST elevation myocardial infarction; CAD, coronary artery disease; MI, myocardial infarction; PCI, percutaneous coronary intervention.

significant predictors and confounders. At this point any variable not selected for the original multivariate model was then added in one at a time, to identify variables that may not be significantly related to the outcome variable but make an important contribution in the presence of other variables. Variables that were significant at the 0.1 level were then included in the model, and the model was again iteratively reduced but only for the variables that were added at the end. Statistical significance was defined as a 2 -sided $\mathrm{P}$ values of $<0.05$. All statistical analyses were performed with the use of STATA software (version 15, StataCorp 2017). Data is reported according to the number of data points available. We do not use statistical techniques, such as imputation, to replace missing data with substituted values. We also do not default missing values to any category.

\section{Results}

\section{Patient characteristics}

We identified a total of 3,722 consecutive index admissions for NSTEMI where coronary angiography was performed and there was no prior CABG. Of these, 1,278 (34\%) had 1-VD, 793 (21\%) had 2-VD and 769 (21\%) had 3-VD, meaning that $1,562(42 \%)$ had MVD. 883 (24\%) patients had $0-V D$. The patient characteristics for each subgroup are presented in Table 1. The $0-\mathrm{VD}$ group were mainly female while all other groups were predominantly male. An increasing number of significantly diseased vessels was associated with older age, a history of diabetes mellitus, hypertension, dyslipidaemia, smoking and prior MI. The number of traditional atherosclerotic risk factors present increased with the number of diseased vessels. Notably only $3.2 \%$ of $1-\mathrm{VD}, 2.4 \%$ of $2-\mathrm{VD}$ and $3.1 \%$ of $3-\mathrm{VD}$ patients had no traditional risk factors recorded, compared to $7.9 \%$ of patients with $0-\mathrm{VD}(\mathrm{P}<0.001)$ (Figure 2).

Characteristics of patients with MVD in comparison to patients without MVD are shown in Table 2. A multivariate logistic regression model showed that age, male gender, diabetes, dyslipidaemia and prior MI all independently predicted MVD over and above having single vessel or no 
Prevalence of risk factors by number of affected vessels

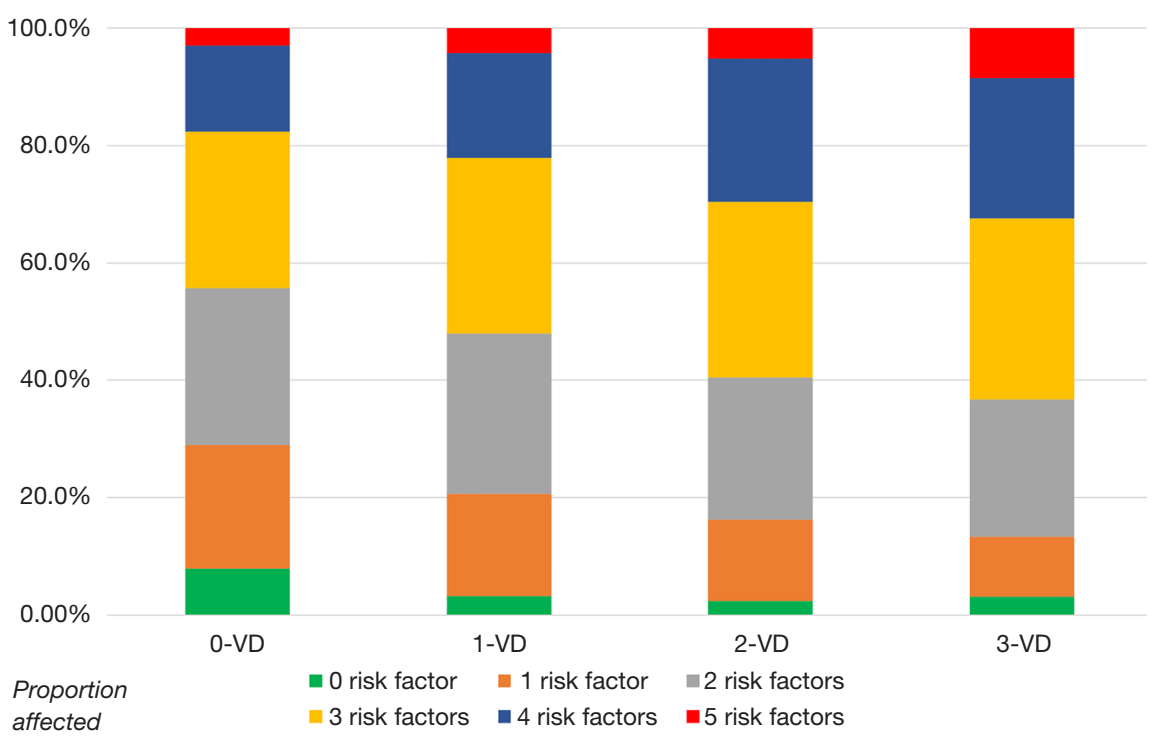

Figure 2 Prevalence of traditional cardiovascular risk factors. Graph shows the number of traditional cardiovascular risk factors presented by the number of severely stenosed vessels.

Table 2 Characteristics of NSTEMI patients categorised by the presence or absence of multi-vessel coronary artery disease

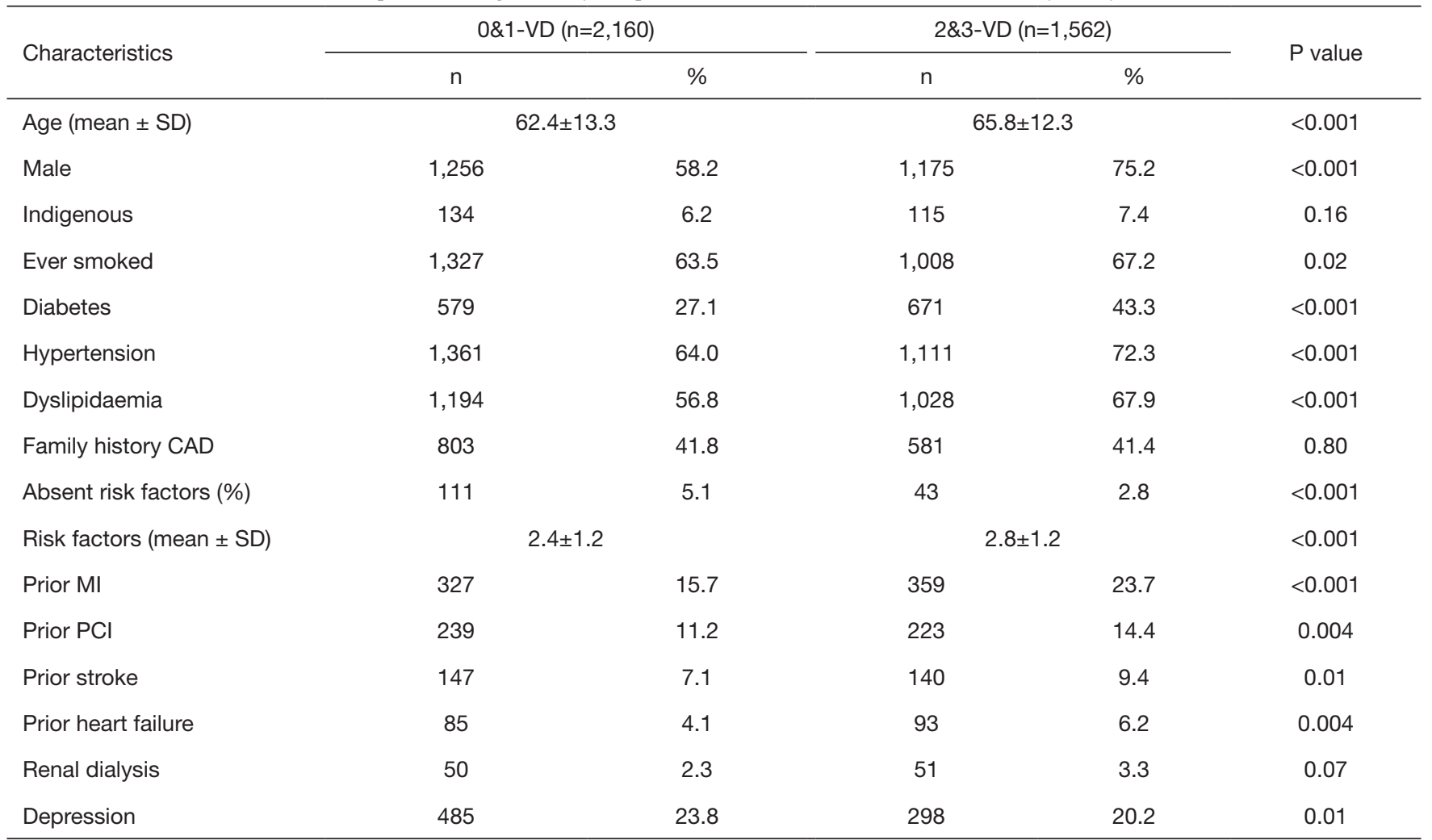

NSTEMI, non-ST elevation myocardial infarction; CAD, coronary artery disease; MI, myocardial infarction; PCl, percutaneous coronary intervention. 
Table 3 Multivariate logistic regression model of predictors of MVD over zero or one vessel disease

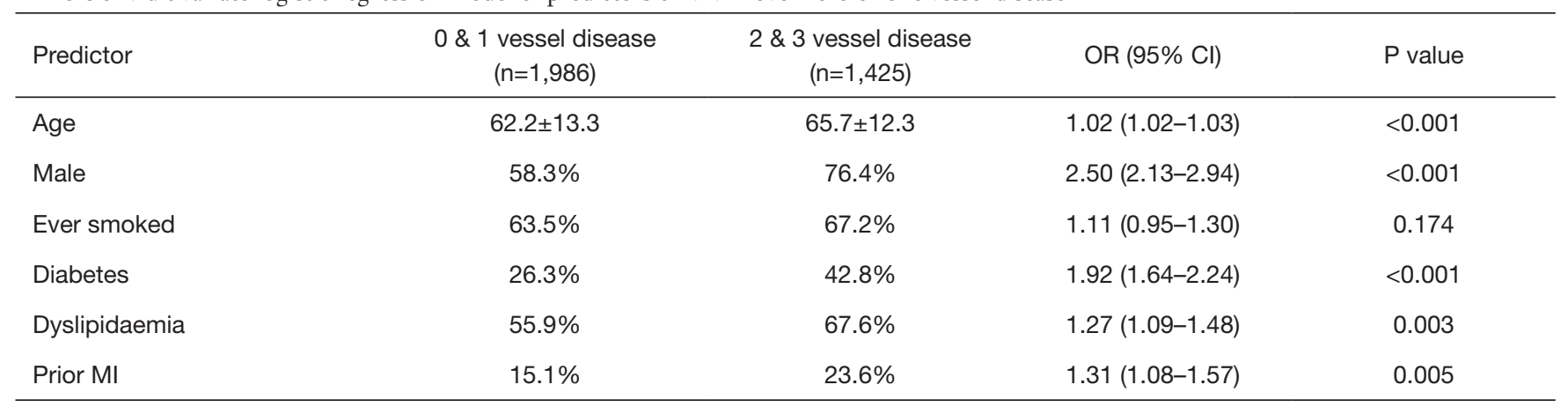

MVD, multivessel coronary disease. MI, myocardial infarction.

significant coronary artery stenosis (Table 3).

The distribution of angiographically-defined coronary artery narrowings is shown in Table 4 . In patients with at least $1-\mathrm{VD}$, the LAD was the most commonly involved vessel, with similar rates of severe stenosis found in the $\mathrm{LCx}$ and RCA. Angiographically significant left main disease was present in only $3 \%$ of patients with $2-\mathrm{VD}$, but in $229(30 \%)$ of those with $3-\mathrm{VD}$.

\section{Management and in-hospital mortality}

The most common recommendations for management were PCI in the 1-VD and $2-\mathrm{VD}$ groups $(69 \%$ and $55 \%$ respectively) and CABG in the $3-\mathrm{VD}$ group $(66 \%)$ (Figure 3A). PCI was performed in 649 out of $1,562(42 \%)$ patients with MVD, including 482 of 793 (61\%) patients with 2-VD and 167 of 769 (22\%) patients with 3-VD (Figure 3B). Of the 649 patients with MVD who underwent PCI, 491 (76\%) received single-vessel PCI alone. In those with 2-VD receiving PCI, 388 patients $(81 \%)$ had singlevessel PCI, while only 92 (19\%) had PCI to two vessels. Two patients in the 2-VD group had PCI to three vessels, due to stenting of an angiographically moderate lesion, with or without FFR guidance. In patients with 3-VD receiving PCI, 103 patients (62\%) had single-vessel PCI, 45 (27\%) had two-vessel PCI and only 19 (11\%) had PCI to all three coronary artery territories.

With respect to staged procedures, 546 patients with MVD had PCI during their initial procedure. In this cohort, repeat procedures were performed in $38(7.0 \%)$ patients, 21 (4.0\%) during the index admission and $16(2.9 \%)$ beyond discharge at an average of $15 \pm 21$ days. Of patients having a second procedure, $32(84.2 \%)$ were planned and 31 (81.6\%) were non-culprit interventions. No patient in the entire studied cohort had three procedures within 90 days, thus excluding 1,016 patients with MVD who had an initial coronary angiogram without PCI.

During the index admission, 35 (0.9\%) patients died with in-hospital mortality increasing in concordance with the number of diseased vessels $(0.3 \%$ for $0-\mathrm{VD}, 0.5 \%$ for $1-\mathrm{VD}$, $1.3 \%$ for $2-\mathrm{VD}$ and $2.0 \%$ for $3-\mathrm{VD}, \mathrm{P}=0.002$ ).

\section{Discussion}

This retrospectively analysed contemporary registry study found that the prevalence of MVD in patients undergoing coronary angiography for NSTEMI was $42 \%$. A previous Portuguese study reported a prevalence of $18 \%$ for MVD (13), but others have observed a similar prevalence of $40-60 \%$ in non-consecutive patients with NSTEMI who are selected for management by PCI (14-16). Although underpowered to determine the effect of MVD on clinical outcomes, our data suggest that it is associated with higher inpatient mortality, a finding well established with respect to all acute coronary syndromes (17).

Whereas complete revascularisation has been shown to be beneficial in prospective randomised STEMI trials (18), it is still unclear whether this strategy is of benefit in NSTEMI (8). Recent large retrospective studies have shown a reduction in long-term all-cause mortality with single-session, multivessel (complete) PCI compared with culprit-lesion PCI alone $(15,19)$. Our study has shown that MVD is prevalent among Australian NSTEMI patients but that where PCI is performed, revascularisation to all significantly diseased vessels occurred in only $19 \%$ of $2-\mathrm{VD}$ and $11 \%$ of 3 -VD patients. It should be noted our data was collected before the seminal COMPLETE trial showed benefit for multi-vessel PCI in the STEMI 


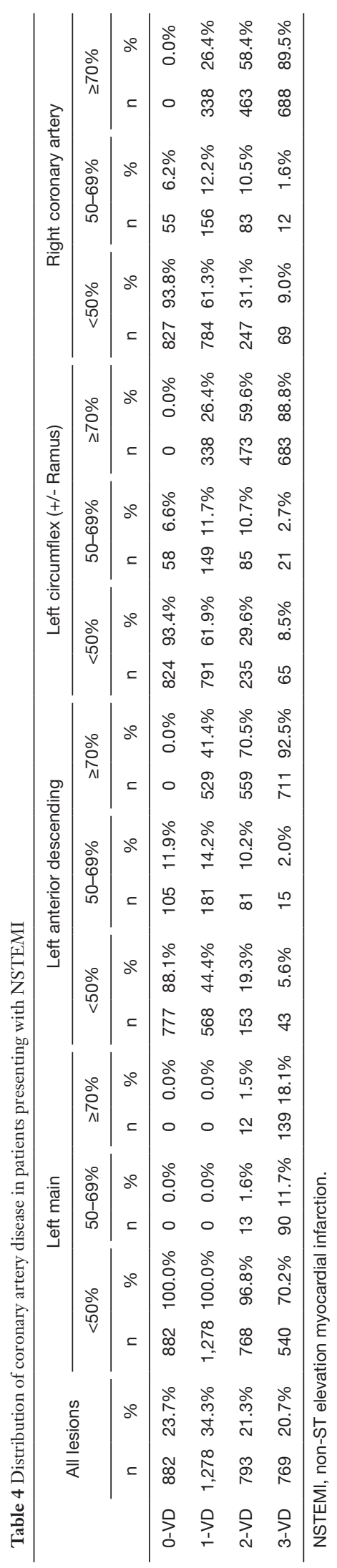

population, which in turn may have influenced practice in the NSTEMI cohort (18). We have shown a small number of patients with MVD undergoing PCI in their index procedure had a second PCI procedure, either during the index admission or within 90 days of discharge. Most of these procedures were planned interventions on non-culprit lesions. The overall numbers are small in keeping with a paucity of evidence supporting non-culprit intervention in NSTEMI (8). However, whether the second procedure represented complete revascularization is unclear. This is an area requiring further examination in future trials with data pertaining to both real world practice elsewhere and outcomes lacking. As expected, we found that the proportion of patients with NSTEMI referred for CABG is higher in those with $3-\mathrm{VD}$ than 2-VD. However, it is unclear from this analysis in how many this represented complete revascularisation. More prospective data are required to inform optimal revascularisation strategies in this important and common clinical setting.

A renewed focus on secondary prevention measures in patients with NSTEMI and MVD is also required. We observed that the number of traditional risk factors for atherosclerosis increases with the number of diseased vessels and that increasing age, male gender, diabetes, dyslipidaemia and prior AMI are all independently associated with the presence of MVD over 0-VD or 1-VD. This underscores the importance of risk factor control. A small number of patients had MVD in the absence of traditional risk factors, highlighting the possible influence of under-recognised contributors to coronary disease. Several recent trials have examined systemic anti-inflammatory therapy in preventing atherosclerotic events with mixed results $(20,21)$. Recent trials of colchicine are promising, though overall mortality benefit is elusive $(22,23)$. A minority of MVD patients in our study were treated with medical management alone but should future trials of novel medical therapies in this cohort prove beneficial, this may lessen the need for multivessel or complete revascularisation.

Interestingly, we also identified that almost a quarter of NSTEMI patients had 0-VD, with most appropriately treated medically, consistent with a lack of evidence to support percutaneous stenting of non-severe plaques (24). The cohort of 0-VD patients would have included, but would not be limited to, patients with myocardial infarction with nonobstructive coronary arteries (MINOCA), defined as patients with an AMI but with no obstructive lesion >50\% in severity and no clinically apparent diagnosis (25). The prevalence of MINOCA amongst cohorts of patients with 
A

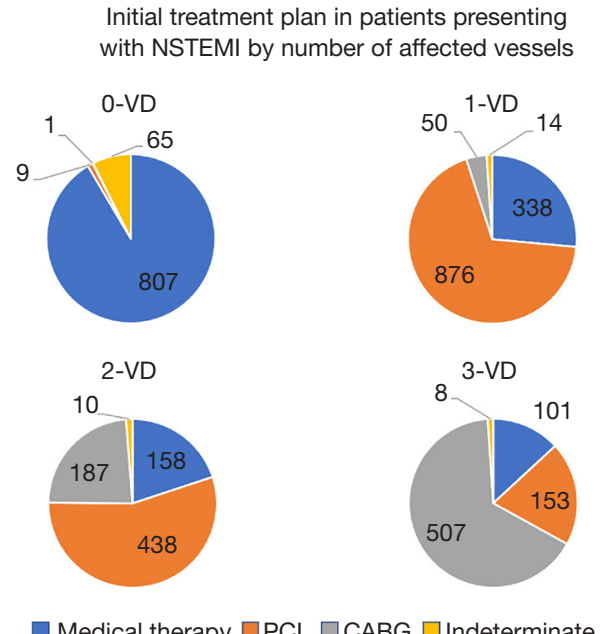

B
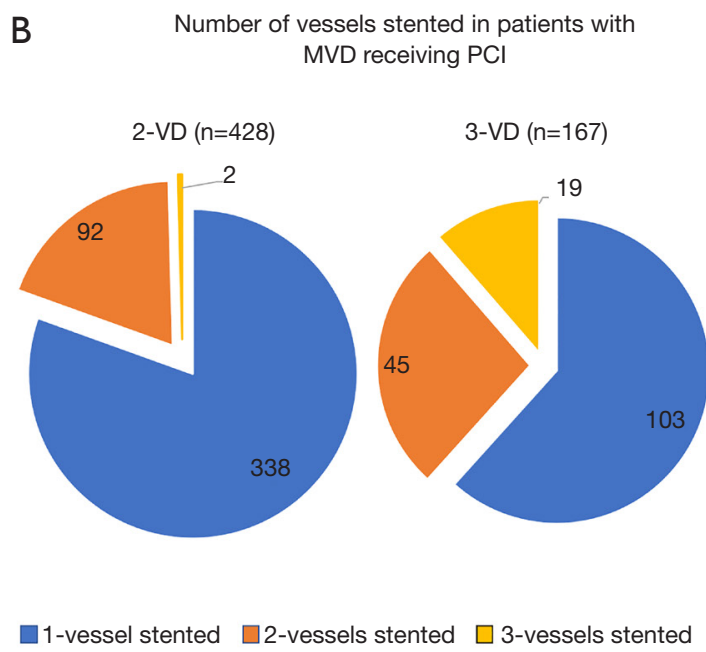

Figure 3 Inpatient management of MVD in NSTEMI. (A) Pie-charts show the initial treatment plan by number of severely stenosed vessels. (B) Pie-charts show the number of vessels stented among patients treated with percutaneous coronary intervention in the 2-VD and 3-VD groups. NSTEMI, non-ST elevation myocardial infarction; MVD, multivessel coronary disease. VD, vessel disease; PCI, percutaneous coronary intervention.

AMI has been estimated to be approximately $6 \%$ (26) but is more frequent in NSTEMI, where it rises to $8-10 \%(27,28)$. In addition, the $0-\mathrm{VD}$ group in our study also included patients with at worst angiographically moderate stenoses of $50-69 \%$ severity. Finally, other non-atherosclerotic causes of NSTEMI, such as coronary vasospasm or spontaneous coronary dissection would also have been captured. This is reflected by the lower prevalence of traditional risk factors for atherosclerosis, younger age and female predominance $(57.6 \%)$ that we observed in the $0-\mathrm{VD}$ group. Taken together, the relatively high prevalence of $0-\mathrm{VD}$ in our study highlights the heterogenous basis of NSTEMI and underscores the necessity that future research addresses the fundamental pathophysiological mechanism in each individual patient.

Unsurprisingly, we have demonstrated inpatient mortality increases significantly with the number of severely stenosed vessels. However, it should be noted that the inpatient mortality rate we observed is lower than that described in previous studies of NSTEMI cohorts, in part due to a younger population $(29,30)$. Furthermore, as our study only included patients undergoing angiography, it likely excluded many elderly, frail or co-morbid patients who would likely have been deemed inappropriate for coronary angiography because of their high risk, poor quality of life or poor prognosis. Inclusion of these patients in an all-comers registry would have almost certainly resulted in a higher mortality rate. As a comparison, one recent study that included all patients presenting with NSTEMI, only $80 \%$ of whom underwent angiography, reported 30-day inpatient mortality of $6.29 \%$ (30). Another study included not only patients undergoing angiography but also required them to have undergone PCI (29), thus excluding patients with $0-\mathrm{VD}$. This would explain their higher inpatient mortality rate of $2.0 \%$, given that patients with $0-\mathrm{VD}$ have a more favorable inpatient prognosis, as revealed by our own data.

The CADOSA registry is a comprehensive clinical quality registry with extensive details, but since data collection was not prospectively designed to address some of the variables of interest for this study, there are some limitations. Thus, while angiographic lesion severity and risk factors were captured, the characteristics of the lesions and the severity of individual risk factors were not. Furthermore, data pertaining to whether CABG or multivessel PCI constituted 'complete' revascularisation were not captured, nor were long term outcomes.

\section{Conclusions}

MVD in NSTEMI has a prevalence of approximately $40 \%$ in a real-world population. In patients undergoing PCI, culprit-lesion only revascularisation during the index admission has been preferred in our practice. As the question of whether multivessel PCI confers superior 
outcomes in these patients remains largely unanswered, future prospective trials are needed to investigate optimal revascularisation strategies.

\section{Acknowledgments}

Funding: CADOSA is supported by South Australian Cardiovascular Research Development Program (SACVRDP) funded by the Heart Foundation of South Australia and South Australian Government. CADOSA is also supported by The University of Adelaide Research Infrastructure Scheme. PJP is supported by research fellowships from the National Heart Foundation of Australia (Future Leader Fellowship FLF102056) and National Health and Medical Research Council of Australia (CDF1161506).

\section{Footnote}

Reporting Checklist: The authors have completed the STROBE reporting checklist. Available at https://cdt. amegroups.com/article/view/10.21037/cdt-21-518/rc

Data Sharing Statement: Available at https://cdt.amegroups. com/article/view/10.21037/cdt-21-518/dss

Peer Review File: Available at https://cdt.amegroups.com/ article/view/10.21037/cdt-21-518/prf

Conflicts of Interest: All authors have completed the ICMJE uniform disclosure form (available at https://cdt.amegroups. com/article/view/10.21037/cdt-21-518/coif). PJP has received speaker honoraria ad hoc from Astra Zeneca and Boehringer Ingelheim related to antiplatelet/anticoagulant management of coronary syndromes, and support from Astra Zeneca to attend American Heart Association meeting in 2019. All other grant, advisory committee and honoraria support are not related to the content of this manuscript. The other authors have no conflicts of interest to declare.

Ethical Statement: The authors are accountable for all aspects of the work in ensuring that questions related to the accuracy or integrity of any part of the work are appropriately investigated and resolved. The study was conducted in accordance with the Declaration of Helsinki (as revised in 2013). The study was approved by Central
Adelaide Local Health Network Human Ethics Committee (HREC reference number HREC/15/TQEH/252) and individual consent for this retrospective analysis was waived.

Open Access Statement: This is an Open Access article distributed in accordance with the Creative Commons Attribution-NonCommercial-NoDerivs 4.0 International License (CC BY-NC-ND 4.0), which permits the noncommercial replication and distribution of the article with the strict proviso that no changes or edits are made and the original work is properly cited (including links to both the formal publication through the relevant DOI and the license). See: https://creativecommons.org/licenses/by-nc-nd/4.0/.

\section{References}

1. Moran AE, Forouzanfar MH, Roth GA, et al. Temporal trends in ischemic heart disease mortality in 21 world regions, 1980 to 2010: the Global Burden of Disease 2010 study. Circulation 2014;129:1483-92.

2. AIHW. Heart, stroke and vascular disease-Australian facts. Canberra: AIHW 2021.

3. Sanchis-Gomar F, Perez-Quilis C, Leischik R, et al. Epidemiology of coronary heart disease and acute coronary syndrome. Ann Transl Med 2016;4:256.

4. Darling CE, Fisher KA, McManus DD, et al. Survival after hospital discharge for ST-segment elevation and non-ST-segment elevation acute myocardial infarction: a population-based study. Clin Epidemiol 2013;5:229-36.

5. Nadlacki B, Horton D, Hossain S, et al. Long term survival after acute myocardial infarction in Australia and New Zealand, 2009-2015: a population cohort study. Med J Aust 2021;214:519-25.

6. Corpus RA, House JA, Marso SP, et al. Multivessel percutaneous coronary intervention in patients with multivessel disease and acute myocardial infarction. Am Heart J 2004;148:493-500.

7. Sorajja P, Gersh BJ, Cox DA, et al. Impact of multivessel disease on reperfusion success and clinical outcomes in patients undergoing primary percutaneous coronary intervention for acute myocardial infarction. Eur Heart J 2007;28:1709-16.

8. Baumann AAW, Mishra A, Worthley MI, et al. Management of multivessel coronary artery disease in patients with non-ST-elevation myocardial infarction: a complex path to precision medicine. Ther Adv Chronic Dis 2020;11:2040622320938527. 
9. Saito Y, Kobayashi Y. Percutaneous coronary intervention strategies in patients with acute myocardial infarction and multivessel disease: Completeness, timing, lesion assessment, and patient status. J Cardiol 2019;74:95-101.

10. Collet JP, Thiele H, Barbato E, et al. 2020 ESC Guidelines for the management of acute coronary syndromes in patients presenting without persistent ST-segment elevation. Eur Heart J 2021;42:1289-367.

11. Amsterdam EA, Wenger NK, Brindis RG, et al. 2014 AHA/ACC guideline for the management of patients with non-ST-elevation acute coronary syndromes: executive summary: a report of the American College of Cardiology/ American Heart Association Task Force on Practice Guidelines. Circulation 2014;130:2354-94.

12. Cannon CP, Battler A, Brindis RG, et al. American College of Cardiology key data elements and definitions for measuring the clinical management and outcomes of patients with acute coronary syndromes. A report of the American College of Cardiology Task Force on Clinical Data Standards (Acute Coronary Syndromes Writing Committee). J Am Coll Cardiol 2001;38:2114-30.

13. Carvalho JF, Belo A, Congo K, et al. Left main and/or three-vessel disease in patients with non-ST-segment elevation myocardial infarction and low-risk GRACE score: Prevalence, clinical outcomes and predictors. Rev Port Cardiol (Engl Ed) 2018;37:911-9.

14. Thiele H, Rach J, Klein N, et al. Optimal timing of invasive angiography in stable non-ST-elevation myocardial infarction: the Leipzig Immediate versus early and late PercutaneouS coronary Intervention triAl in NSTEMI (LIPSIA-NSTEMI Trial). Eur Heart J 2012;33:2035-43.

15. Rathod KS, Koganti S, Jain AK, et al. Complete Versus Culprit-Only Lesion Intervention in Patients With Acute Coronary Syndromes. J Am Coll Cardiol 2018;72:1989-99.

16. Mehta SR, Granger CB, Boden WE, et al. Early versus delayed invasive intervention in acute coronary syndromes. N Engl J Med 2009;360:2165-75.

17. Muller DW, Topol EJ, Ellis SG, et al. Multivessel coronary artery disease: a key predictor of short-term prognosis after reperfusion therapy for acute myocardial infarction. Thrombolysis and Angioplasty in Myocardial Infarction (TAMI) Study Group. Am Heart J 1991;121:1042-9.

18. Mehta SR, Wood DA, Storey RF, et al. Complete Revascularization with Multivessel PCI for Myocardial
Infarction. N Engl J Med 2019;381:1411-21.

19. Kim MC, Hyun JY, Ahn Y, et al. Optimal Revascularization Strategy in Non-ST-Segment-Elevation Myocardial Infarction With Multivessel Coronary Artery Disease: Culprit-Only Versus One-Stage Versus Multistage Revascularization. J Am Heart Assoc 2020;9:e016575.

20. Ridker PM, Everett BM, Thuren T, et al. Antiinflammatory Therapy with Canakinumab for Atherosclerotic Disease. N Engl J Med 2017;377:1119-31.

21. Ridker PM, Everett BM, Pradhan A, et al. Low-Dose Methotrexate for the Prevention of Atherosclerotic Events. N Engl J Med 2019;380:752-62.

22. Tardif JC, Kouz S, Waters DD, et al. Efficacy and Safety of Low-Dose Colchicine after Myocardial Infarction. N Engl J Med 2019;381:2497-505.

23. Nidorf SM, Fiolet ATL, Mosterd A, et al. Colchicine in Patients with Chronic Coronary Disease. N Engl J Med 2020;383:1838-47.

24. Zimmermann FM, Ferrara A, Johnson NP, et al. Deferral vs. performance of percutaneous coronary intervention of functionally non-significant coronary stenosis: 15-year follow-up of the DEFER trial. Eur Heart J 2015;36:3182-8.

25. Agewall S, Beltrame JF, Reynolds HR, et al. ESC working group position paper on myocardial infarction with non-obstructive coronary arteries. Eur Heart J 2017;38:143-53.

26. Pasupathy S, Air T, Dreyer RP, et al. Systematic review of patients presenting with suspected myocardial infarction and nonobstructive coronary arteries. Circulation 2015;131:861-70.

27. Planer D, Mehran R, Ohman EM, et al. Prognosis of patients with non-ST-segment-elevation myocardial infarction and nonobstructive coronary artery disease: propensity-matched analysis from the Acute Catheterization and Urgent Intervention Triage Strategy trial. Circ Cardiovasc Interv 2014;7:285-93.

28. Gehrie ER, Reynolds HR, Chen AY, et al. Characterization and outcomes of women and men with non-ST-segment elevation myocardial infarction and nonobstructive coronary artery disease: results from the Can Rapid Risk Stratification of Unstable Angina Patients Suppress Adverse Outcomes with Early Implementation of the ACC/AHA Guidelines (CRUSADE) quality improvement initiative. Am Heart J 2009;158:688-94.

29. Ozaki Y, Katagiri Y, Onuma Y, et al. CVIT expert consensus document on primary percutaneous coronary 
intervention (PCI) for acute myocardial infarction (AMI)

in 2018. Cardiovasc Interv Ther 2018;33:178-203.

30. Seghieri C, Mimmi S, Lenzi J, et al. 30-day in-hospital

Cite this article as: Baumann AAW, Tavella R, Air TM, Mishra A, Montarello NJ, Arstall M, Zeitz C, Worthley MI, Beltrame JF, Psaltis PJ. Prevalence and real-world management of NSTEMI with multivessel disease. Cardiovasc Diagn Ther 2022;12(1):1-11. doi: 10.21037/cdt-21-518 mortality after acute myocardial infarction in Tuscany (Italy): an observational study using hospital discharge data. BMC Med Res Methodol 2012;12:170. 